\title{
WEIGHTED MIXED WEAK-TYPE INEQUALITIES FOR MULTILINEAR OPERATORS
}

\author{
KANGWEI LI, SHELDY J. OMBROSI, AND M. BELÉN PICARDI
}

\begin{abstract}
In this paper we present a theorem that generalizes Sawyer's classic result about mixed weighted inequalities to the multilinear context. Let $\vec{w}=\left(w_{1}, \ldots, w_{m}\right)$ and $\nu=w_{1}^{\frac{1}{m}} \ldots w_{m}^{\frac{1}{m}}$, the main result of the paper sentences that under different conditions on the weights we can obtain

$$
\left\|\frac{T(\vec{f})(x)}{v}\right\|_{L^{\frac{1}{m}, \infty}\left(\nu v^{\left.\frac{1}{m}\right)}\right.} \leq C \prod_{i=1}^{m}\left\|f_{i}\right\|_{L^{1}\left(w_{i}\right)},
$$

where $T$ is a multilinear Calderón-Zygmund operator. To obtain this result we first prove it for the $m$-fold product of the Hardy-Littlewood maximal operator $M$, and also for $\mathcal{M}(\vec{f})(x)$ : the multi(sub)linear maximal function introduced in [13].

As an application we also prove a vector-valued extension to the mixed weighted weak-type inequalities of multilinear Calderón-Zygmund operators.
\end{abstract}

\section{INTRODUCTION}

In 1985, E. Sawyer [18] proved the following mixed weak-type inequality:

Theorem 1.1. If $u, v \in A_{1}$, then there is a constant $C$ such that for all $t>0$,

$$
u v\left(\left\{x \in \mathbb{R}: \frac{M(f v)(x)}{v(x)}>t\right\}\right) \leq \frac{C}{t} \int_{\mathbb{R}}|f(x)| u(x) v(x) d x .
$$

In the same work E. Sawyer conjectured that the previous theorem is valid if the maximal operator is replaced by the Hilbert transform. In 2005, D. Cruz-Uribe, J. M. Martell and C. Pérez [7] extended this result to $\mathbb{R}^{n}$. Furthermore, they proved it for Calderón-Zygmund operators, solving this Sawyer's conjecture.

Theorem $1.2([7])$. If $u, v \in A_{1}$, or $u \in A_{1}$ and $u v \in A_{\infty}$, then there is a constant $C$ such that for all $t>0$,

$$
u v\left(\left\{x \in \mathbb{R}^{n}: \frac{|T(f v)(x)|}{v(x)}>t\right\}\right) \leq \frac{C}{t} \int_{\mathbb{R}^{n}}|f(x)| u(x) v(x) d x,
$$

where $T$ is Hardy-Littlewood maximal function or any Calderón-Zygmund operator.

Quantitative estimates of these mixed weighted results can be found in [16]. Moreover, it was conjectured in [7] that the conclusion of the previous theorem still holds if a weaker and more general hypothesis is satisfied. That is, if we have the following conditions on the weights, $u \in A_{1}$ and $v \in A_{\infty}$. Recently, in [14] the first two authors and C. Pérez solved such conjecture. Namely, the following theorem was proved, which constitutes the more difficult case of this class of mixed weighted inequalities.

Key words and phrases. mixed weighted inequalities, multilinear operators.

K.L. is supported by Juan de la Cierva-Formación 2015 FJCI-2015-24547, the Basque Government through the BERC 2014-2017 program and Spanish Ministry of Economy and Competitiveness MINECO: BCAM Severo Ochoa excellence accreditation SEV-2013-0323. S. O. and B. P. are supported by CONICET PIP 11220130100329CO, Argentina. 
Theorem 1.3 ([14]). Let $T$ be a Calderón-Zygmund operator or the Hardy-Littlewood maximal operator and let $u \in A_{1}$ and $v \in A_{\infty}$. Then there is a finite constant $C$ depending on the $A_{1}$ constant of $u$ and the $A_{\infty}$ constant of $v$ such that

$$
\left\|\frac{T(f v)}{v}\right\|_{L^{1, \infty}(u v)} \leq C\|f\|_{L^{1}(u v)} .
$$

On the other hand, the study of multilinear Calderón-Zygmund theory started in the seventies with the works of R. Coifman and Y. Meyer ([5] and [6]). However, a systematic treatment of this topic appears later with works of L. Grafakos and R. Torres [11, 12]. We recall the definition of a multilinear Calderón-Zygmund operator: let $T: S\left(\mathbb{R}^{n}\right) \times \cdots \times S\left(\mathbb{R}^{n}\right) \rightarrow S^{\prime}\left(\mathbb{R}^{n}\right)$ be a multilinear operator initially defined on the $m$-fold product of Schwartz spaces and taking values into the space of tempered distributions; we say that $T$ is an $m$-linear Calderón-Zygmund operator if, for some $1 \leq q_{1}, \ldots, q_{m}<\infty$ and $\frac{1}{m} \leq p<\infty$ satisfying $\frac{1}{p}=\frac{1}{q_{1}}+\cdots+\frac{1}{q_{m}}$, it extends to a bounded multilinear operator from $L^{q_{1}} \times \cdots \times L^{q_{m}}$ to $L^{p}$, and if there exists a function $K$ defined off the diagonal $x=y_{1}=\cdots=y_{m}$ in $\left(\mathbb{R}^{n}\right)^{m+1}$ satisfying the appropriate decay and smoothness conditions (see Page 5 in [13] ) and such that

$$
T\left(f_{1}, \ldots, f_{m}\right)(x)=\int_{\mathbb{R}^{n}} \cdots \int_{\mathbb{R}^{n}} K\left(x, y_{1}, \ldots, y_{m}\right) \prod_{i=1}^{m} f_{i}\left(y_{i}\right) d y_{1} \cdots d y_{m}
$$

for all $x \notin \cap_{i=1}^{m} \operatorname{supp} f_{i}$. Related to weighted estimates for these operators, the first result was obtained in [12] (see also [17]) where the authors proved that, if $1<q_{1}, \ldots, q_{m}<\infty$ and $w$ is a weight in the Muckenhoupt $A_{q_{0}}$ class for $q_{0}=\min \left\{q_{1}, \ldots, q_{m}\right\}$, an $m$-linear Calderón-Zygmund operator $T$ maps $L^{q_{1}}(w) \times \cdots \times L^{q_{m}}(w)$ into $L^{p}(w)$. In [13] Lerner et. al, developed the appropriate class of multiple weights for $m$-linear Calderón-Zygmund operators. Now, we recall some of those results in [13] that will be useful for us along this paper. Let $1 \leq q_{1}, \ldots, q_{m}<\infty$ and $\frac{1}{m} \leq p<\infty$ be such that $\frac{1}{p}=\frac{1}{q_{1}}+\cdots+\frac{1}{q_{m}}$. We say that $\vec{w}=\left(w_{1}, \ldots, w_{m}\right)$ satisfies the multilinear $A_{\vec{q}}$ condition if

$$
\sup _{Q}\left(\frac{1}{|Q|} \int_{Q} \nu_{\vec{w}}\right)^{1 / p} \prod_{i=1}^{m}\left(\frac{1}{|Q|} \int_{Q} w_{i}^{1-q_{i}^{\prime}}\right)^{1 / q_{i}^{\prime}}<\infty
$$

where the supremum is taken over all cubes $Q$ (when $q_{i}=1,\left(\frac{1}{|Q|} \int_{Q} w_{i}^{1-q_{i}^{\prime}}\right)^{1 / q_{i}^{\prime}}$ is understood as $\left.\left(\inf _{Q} w_{i}\right)^{-1}\right)$. Now, if $\vec{w}$ satisfies the $A_{\vec{q}}$ condition and $1<q_{1}, \ldots, q_{m}<\infty$, then an $m$-linear Calderón-Zygmund operator $T$ maps $L^{q_{1}}\left(w_{1}\right) \times \cdots \times L^{q_{m}}\left(w_{m}\right)$ into $L^{p}\left(\nu_{\vec{w}}\right)$. If at least one $q_{i}=1$, then $T$ maps $L^{q_{1}}\left(w_{1}\right) \times \cdots \times L^{q_{m}}\left(w_{m}\right)$ into $L^{p, \infty}\left(\nu_{\vec{w}}\right)$. It is shown that $\prod_{i=1}^{m} A_{q_{i}} \subseteq A_{\vec{q}}$ and that this inclusion is strict. Moreover, if $T$ is the $m$-linear Riesz transform, it was proved in [13] that $A_{\vec{q}}$ is a necessary condition for such weighted estimate of $T$.

One of the key points in [13] was the introduction of the multi(sub)linear maximal function $\mathcal{M}$ defined by

$$
\mathcal{M}(\vec{f})(x)=\sup _{Q \ni x} \prod_{i=1}^{m} \frac{1}{|Q|} \int_{Q}\left|f_{i}\left(y_{i}\right)\right| d y_{i},
$$

where $\vec{f}=\left(f_{1}, \ldots, f_{m}\right)$ and the supremum is taken over all cubes $Q$ containing $x$.

This maximal operator is smaller than the product $\prod_{i=1}^{m} M f_{i}$, which was the auxiliary operator used previously to estimate multilinear singular integral operators.

The aim of this paper is to obtain mixed weighted estimates that generalize Theorem 1.3 to the multilinear context. We will investigate both $\prod_{i=1}^{m} M f_{i}$ and $\mathcal{M}(\vec{f})$ under different assumptions. Then by an extrapolation theorem we can also prove mixed weighted inequalities for multilinear Calderón-Zygmund operators. 
The first result of this paper is the following:

Theorem 1.4. Let $w_{1}, \ldots, w_{m} \in A_{1}$ and $v \in A_{\infty}$. Denote $\nu=w_{1}^{\frac{1}{m}} \ldots w_{m}^{\frac{1}{m}}$. Then,

$$
\left\|\frac{\prod_{i=1}^{m} M f_{i}}{v}\right\|_{L^{\frac{1}{m}, \infty}\left(\nu v^{\frac{1}{m}}\right)} \leq C \prod_{i=1}^{m}\left\|f_{i}\right\|_{L^{1}\left(w_{i}\right)} .
$$

The particular case in which the weight $v=1$ in the theorem above was proved in [13] (See Theorem 3.12 there). Adding a non-constant function $v$ in the distribution function makes the proof more complicated. However, benefits from Theorem 1.3 and the ideas in [13] allow us to obtain the result. It is obvious that the conclusion in Theorem 1.4 also holds for the maximal operator $\mathcal{M}$.

We will see below that as a consequence of Theorem 1.4, we can obtain the same result for multilinear Calderón-Zygmund operators. However, we know that $\prod_{i=1}^{m} M f_{i}$ is too big to estimate multilinear Calderón-Zygmund operators. In fact, it was proved in [13] that the condition $w_{1}, \ldots, w_{m} \in A_{1}$ is stronger than $\vec{w}=\left(w_{1}, \ldots, w_{m}\right) \in A_{\overrightarrow{1}}$. And since the last condition characterizes the weak type of a multilinear Calderón-Zygmund operator $T$ from $L^{1}\left(w_{1}\right) \times \cdots \times$ $L^{1}\left(w_{m}\right)$ into $L^{1 / m, \infty}(\nu)$, it is natural to ask if it is possible to relax the hypothesis $w_{1}, \ldots, w_{m} \in A_{1}$ in Theorem 1.4 if we put $T$ or $\mathcal{M}$ instead of $\prod_{i=1}^{m} M f_{i}$. The next theorem gives a partially positive answer.

Theorem 1.5. Let $\vec{w}=\left(w_{1}, \ldots, w_{m}\right) \in A_{\overrightarrow{1}}, \nu=w_{1}^{\frac{1}{m}} \ldots w_{m}^{\frac{1}{m}}$ and $v$ be a weight satisfying $\nu v^{\frac{1}{m}} \in$ $A_{\infty}$. Then there is a constant $C$ such that

$$
\left\|\frac{\mathcal{M}(\vec{f})(x)}{v}\right\|_{L^{\frac{1}{m}, \infty}\left(\nu v^{\frac{1}{m}}\right)} \leq C \prod_{i=1}^{m}\left\|f_{i}\right\|_{L^{1}\left(w_{i}\right)} .
$$

So (1.6) holds for either $\vec{w}=\left(w_{1}, \ldots, w_{m}\right) \in A_{\overrightarrow{1}}$ and $\nu v^{\frac{1}{m}} \in A_{\infty}$ or (as a consequence of Theorem 1.4) if the weights $w_{i} \in A_{1}$ for $i=1, \ldots, m$ and $v \in A_{\infty}$. These conditions are independent. However, we believe that there is a unified condition that contains both such that (1.6) holds. That is:

Conjecture 1.7. Let $\vec{w}=\left(w_{1}, \ldots, w_{m}\right) \in A_{\overrightarrow{1}}, v^{1 / m} \in A_{\infty}$ and $\nu=w_{1}^{\frac{1}{m}} \ldots w_{m}^{\frac{1}{m}}$. Then there is $a$ constant $C$ such that

$$
\left\|\frac{\mathcal{M}(\vec{f})(x)}{v}\right\|_{L^{\frac{1}{m}, \infty}\left(\nu v^{\frac{1}{m}}\right)} \leq C \prod_{i=1}^{m}\left\|f_{i}\right\|_{L^{1}\left(w_{i}\right)}
$$

Remark 1.8. In general, under the hypothesis of Theorem 1.5 the estimate (1.6) does not hold if $\mathcal{M}(\vec{f})$ is replaced by $\prod_{i=1}^{m} M f_{i}$ even in the case that $v(x)=1$. This fact was proved in Remark 7.5 in [13].

The following (extrapolation) theorem allows us to reduce the problem of multilinear CalderónZygmund operators to the multilinear maximal function, exactly as in the linear case. Actually, the theorem below was essentially obtained in [15], which is a combination of Theorem 1.5 and some observations in Section 2.2 there. However, for the sake of completeness we will give a complete proof in Appendix A .

Theorem $1.9([15])$. Let $\vec{w}=\left(w_{1}, \ldots, w_{m}\right) \in A_{\overrightarrow{1}}, v^{1 / m} \in A_{\infty}$ and $\nu=w_{1}^{\frac{1}{m}} \ldots w_{m}^{\frac{1}{m}}$. Then

$$
\left\|\frac{T(\vec{f})}{v}\right\|_{L^{\frac{1}{m}, \infty}\left(\nu v^{\left.\frac{1}{m}\right)}\right.} \leq C\left\|\frac{\mathcal{M}(\vec{f})}{v}\right\|_{L^{\frac{1}{m}, \infty}\left(\nu v^{\frac{1}{m}}\right)}
$$

where $C$ is a constant and $T$ is a multilinear Calderón-Zygmund operator. 
Now, as a consequence of Theorems 1.4, 1.5 and 1.9 we obtain the main result of this paper:

Theorem 1.10. Let $T$ be a multilinear Calderón-Zygmund operator, $\vec{w}=\left(w_{1}, \ldots, w_{m}\right)$ and $\nu=w_{1}^{\frac{1}{m}} \ldots w_{m}^{\frac{1}{m}}$. Suppose that $\vec{w} \in A_{\overrightarrow{1}}$ and $\nu v^{\frac{1}{m}} \in A_{\infty}$ or $w_{1}, \ldots, w_{m} \in A_{1}$ and $v \in A_{\infty}$. Then there is a constant $C$ such that

$$
\left\|\frac{T(\vec{f})(x)}{v}\right\|_{L^{\frac{1}{m}, \infty}\left(\nu v^{\frac{1}{m}}\right)} \leq C \prod_{i=1}^{m}\left\|f_{i}\right\|_{L^{1}\left(w_{i}\right)}
$$

Recall the definition of $R H_{\infty}$ :

Definition 1.11. We denote by $R H_{\infty}$ the class of weights $w$ such that for all cube $Q$, there exists a constant $C$, which is independent of $Q$, such that

$$
\underset{x \in Q}{\operatorname{ess} \sup } w(x) \leq \frac{C}{|Q|} \int_{Q} w(x) d x .
$$

Since if $u \in A_{1}$ and $v \in R H_{\infty}$, then $u v^{\frac{1}{m}} \in A_{\infty}$ (see Lemma 2.1 below), we have a direct corollary of Theorem 1.10.

Corollary 1.12. Let $\vec{w}=\left(w_{1}, \ldots, w_{m}\right) \in A_{\overrightarrow{1}}$ and let $v \in R H_{\infty}$. Then there is a constant $C$ such that

$$
\left\|\frac{T(\vec{f})(x)}{v}\right\|_{L^{\frac{1}{m}, \infty}\left(\nu v^{\frac{1}{m}}\right)} \leq C \prod_{i=1}^{m}\left\|f_{i}\right\|_{L^{1}\left(w_{i}\right)}
$$

The article is organized as follows. In Section 2 we prove Theorem 1.4. The proof of Theorem 1.5 is presented in Section 3. In Section 4 as an application of Theorem 1.10 we obtain a vectorvalued extension of the mixed weighted inequalities obtained for multilinear Calderón-Zygmund operators. The last Section is the Appendix A where we give a complete proof of Theorem 1.9.

\section{Proof of Theorem 1.4}

First, we need the following Lemma.

\section{Lemma 2.1.}

(a) $w \in A_{\infty}$ if and only if $w=w_{1} w_{2}$, where $w_{1} \in A_{1}$ and $w_{2} \in R H_{\infty}$.

(b) If $w \in A_{1}$, then $w^{-1} \in R H_{\infty}$.

(c) If $u, v \in R H_{\infty}$, then $u v \in R H_{\infty}$.

(d) If $w \in A_{\infty}$ and $u \in R H_{\infty}$, then $w u \in A_{\infty}$.

(e) If $w \in R H_{\infty}$, then $w^{s} \in R H_{\infty}$ for any $s>0$.

All these properties of $A_{p}$ classes of Muckenhoupt are well known. The first three can be found in [8] or [9] for instance. However, as far as we know, (d) and (e) are not written specifically in any place, so in the following paragraph we present a simple argument for them.

Proof of (d): Since $w \in A_{\infty}$, by (a), $w=w_{1} w_{2}$, where $w_{1} \in A_{1}$ and $w_{2} \in R H_{\infty}$. By (c), $w_{2} u \in R H_{\infty}$. Then, $w u=\left(w_{1} w_{2}\right) u=w_{1}\left(w_{2} u\right)$. Now, by (a), $w u \in A_{\infty}$.

Proof of (e): If $s \geq 1$, this is just by Hölder's inequality, so we only need to consider the case $s<1$. Since $w \in R H_{\infty} \subset A_{\infty}$, then

$$
\frac{1}{|Q|} \int_{Q} w \leq C_{s, w}\left(\frac{1}{|Q|} \int_{Q} w^{s}\right)^{\frac{1}{s}} .
$$

By definition, our claim follows immediately. 
Proof of Theorem 1.4 : The main idea of this proof is to reduce the problem to the linear case and then apply Theorem 1.3. We define

$$
E=\left\{x: v(x)<\prod_{i=1}^{m} M f_{i}(x) \leq 2 v(x)\right\}
$$

Let $\tilde{v}_{i}=\prod_{j=1, j \neq i}^{m}\left(M f_{j}\right)^{-1}$ and let $v_{i}=v \tilde{v_{i}}$. Observe that $v \in A_{\infty}$ and $\tilde{v}_{i} \in R H_{\infty}$. By Lemma 2.1 (d), $v_{i} \in A_{\infty}$. In order to prove the theorem it is enough to show that

$$
v^{\frac{1}{m}} \nu(E) \leq C \prod_{i=1}^{m}\left\|f_{i}\right\|_{L^{1}\left(w_{i}\right)} .
$$

By Hölder's inequality and Theorem 1.3, we have

$$
\begin{aligned}
v^{\frac{1}{m}} \nu(E) & \leq \int_{E}\left(\prod_{i=1}^{m} M f_{i} w_{i}\right)^{\frac{1}{m}} \leq \prod_{i=1}^{m}\left(\int_{E} M f_{i} w_{i}\right)^{\frac{1}{m}} \leq 2 \prod_{i=1}^{m}\left(\int_{E} v_{i} w_{i}\right)^{\frac{1}{m}} \\
& \leq 2 \prod_{i=1}^{m}\left(\int_{\left\{x: M f_{i}>v_{i}\right\}} v_{i} w_{i}\right)^{\frac{1}{m}} \leq C \prod_{i=1}^{m}\left\|f_{i}\right\|_{L^{1}\left(w_{i}\right)}^{\frac{1}{m}},
\end{aligned}
$$

where in the last inequality we have used Theorem 1.3 since $w_{i} \in A_{1}$ and $v_{i} \in A_{\infty}$.

\section{Proof of Theorem 1.5}

We follow the strategy of [14]. So we only need to consider the dyadic multilinear maximal functions. First, recall that if $\vec{w}=\left(w_{1}, \ldots, w_{m}\right) \in A_{\overrightarrow{1}}$, then $\nu=w_{1}^{\frac{1}{m}} \ldots w_{m}^{\frac{1}{m}} \in A_{1}$ (see Theorem 3.6 in [13]). On the other hand, since $\nu \in A_{1}$ it is not difficult to check that the hypothesis $\nu v^{\frac{1}{m}} \in A_{\infty}$ implies that $v^{\frac{1}{m}} \in A_{\infty}$.

We shall prove

$$
\nu v^{\frac{1}{m}}\left(\left\{x: 1<\frac{\mathcal{M}_{d}\left(f_{1}, \cdots, f_{m}\right)(x)}{v(x)} \leq 2\right\}\right) \leq C\left(\prod_{i=1}^{m} \int_{\mathbb{R}^{n}}\left|f_{i}\right| w_{i}\right)^{\frac{1}{m}}
$$

Without loss of generality, we can assume $f_{i} \geq 0, i=1, \cdots, m$. Let

$$
E_{k}:=\left\{x: 1<\frac{\mathcal{M}_{d}\left(f_{1}, \cdots, f_{m}\right)(x)}{v(x)} \leq 2, a^{m k}<v(x) \leq a^{m(k+1)}\right\},
$$

where $a>2^{n}$. Again, define

$$
\Omega_{k}=\left\{\mathcal{M}_{d}\left(f_{1}, \cdots, f_{m}\right)>a^{m k}\right\}
$$

and let $\left\{I_{j}^{k}\right\}_{j}$ be the collection of maximal dyadic cubes in $\Omega_{k}$. Then by maximality, $a^{m k}<$ $\prod_{i=1}^{m}\left\langle f_{i}\right\rangle_{I_{j}^{k}} \leq 2^{m n} a^{m k}$. Splitting the collection $\left\{I_{j}^{k}\right\}_{j}$ to

$$
\mathcal{Q}_{l, k}=\left\{I_{j}^{k}: a^{k+l} \leq\left\langle v^{\frac{1}{m}}\right\rangle_{I_{j}^{k}}<a^{k+l+1}\right\}, \quad l \in \mathbb{Z} .
$$

Then we have

$$
\begin{aligned}
\sum_{k \in \mathbb{Z}} \nu v^{\frac{1}{m}}\left(E_{k}\right) & =\sum_{k \in \mathbb{Z}} \nu v^{\frac{1}{m}}\left(E_{k} \cap \Omega_{k}\right)=\sum_{k \in \mathbb{Z}} \sum_{j} \nu v^{\frac{1}{m}}\left(E_{k} \cap I_{j}^{k}\right) \\
& \leq \sum_{k \in \mathbb{Z}} \sum_{l \geq 0} \sum_{I_{j}^{k} \in \mathcal{Q}_{l, k}} a^{k+1} \nu\left(E_{k} \cap I_{j}^{k}\right) \\
& =\sum_{k \in \mathbb{Z}} \sum_{l \geq 0} \sum_{I_{j}^{k} \in \Gamma_{l, k}} a^{k+1} \nu\left(E_{k} \cap I_{j}^{k}\right)
\end{aligned}
$$

where

$$
\Gamma_{l, k}=\left\{I_{j}^{k} \in \mathcal{Q}_{l, k}:\left|I_{j}^{k} \cap\left\{x: a^{k}<v^{\frac{1}{m}} \leq a^{k+1}\right\}\right|>0\right\} .
$$


From now on, we shall deal with the case $l=-1$ and $l \geq 0$ separately. By monotone convergence theorem, it suffices to give a uniform estimate for

$$
\sum_{k \geq N} \sum_{l \geq 0} \sum_{I_{j}^{k} \in \Gamma_{l, k}} a^{k+1} \nu\left(E_{k} \cap I_{j}^{k}\right),
$$

where $N<0$. We have the following two lemmas. The proofs are essentially given in [14].

Lemma 3.1. $\Gamma=\cup_{l \in \mathbb{Z}} \cup_{k \geq N} \Gamma_{l, k}$ is sparse.

Lemma 3.2. For $l \geq 0$ and $I_{j}^{k} \in \Gamma_{l, k}$, there exist constants $c_{1}$ and $c_{2}$ depending on $\nu, v$ such that

$$
\nu\left(E_{k} \cap I_{j}^{k}\right) \leq c_{1} e^{-c_{2} l} \nu\left(I_{j}^{k}\right) .
$$

We also have the following lemma.

Lemma 3.3. If $w_{1} w_{2} \in A_{\infty}$, then for any cube $Q$, we have

$$
\left\langle w_{1} w_{2}\right\rangle_{Q} \leq C\left(\left[w_{1} w_{2}\right]_{A_{\infty}}\right)\left\langle w_{1}\right\rangle_{Q}\left\langle w_{2}\right\rangle_{Q}
$$

Proof. let $E_{1}=\left\{x \in Q: w_{1}(x)>4\left\langle w_{1}\right\rangle_{Q}\right\}$ and $E_{2}=\left\{x \in Q: w_{2}(x)>4\left\langle w_{2}\right\rangle_{Q}\right\}$. Then by Chebyshev, it is easy to see that $E:=Q \backslash\left(E_{1} \cup E_{2}\right)$ satisfies $|E| \geq \frac{1}{2}|Q|$. Since $w_{1} w_{2} \in A_{\infty}$, we have

$$
\begin{aligned}
w_{1} w_{2}(Q) & \leq c\left(\left[w_{1} w_{2}\right]_{A_{\infty}}\right) w_{1} w_{2}(E) \leq 16 c\left(\left[w_{1} w_{2}\right]_{A_{\infty}}\right)\left\langle w_{1}\right\rangle_{Q}\left\langle w_{2}\right\rangle_{Q}|E| \\
& \leq 16 c\left(\left[w_{1} w_{2}\right]_{A_{\infty}}\right)\left\langle w_{1}\right\rangle_{Q}\left\langle w_{2}\right\rangle_{Q}|Q|
\end{aligned}
$$

With this lemma, we can also obtain the exponential decay for $l<0$.

Lemma 3.4. For $l<0$ and $I_{j}^{k} \in \Gamma_{l, k}$, there exists a constant $c_{1}$ depending on $\nu, v$ such that

$$
\nu\left(E_{k} \cap I_{j}^{k}\right) \leq c_{1} a^{l} \nu\left(I_{j}^{k}\right)
$$

Proof. By Lemma 3.3, we have

$$
\nu v^{\frac{1}{m}}\left(I_{j}^{k}\right) \leq C_{\nu, v}\langle\nu\rangle_{I_{j}^{k}}\left\langle v^{\frac{1}{m}}\right\rangle_{I_{j}^{k}}\left|I_{j}^{k}\right| \leq C_{\nu, v} a^{k+l} \nu\left(I_{j}^{k}\right)
$$

On the other hand,

Therefore

$$
\nu v^{\frac{1}{m}}\left(I_{j}^{k}\right) \geq a^{k} \nu\left(E_{k} \cap I_{j}^{k}\right)
$$

$$
\nu\left(E_{k} \cap I_{j}^{k}\right) \leq C_{\nu, v} a^{l} \nu\left(I_{j}^{k}\right)
$$

Now fix $l$, form the principal cubes for $\cup_{k \geq N} \Gamma_{l, k}$ : let $\mathcal{P}_{0}^{l}$ be the maximal cubes in $\cup_{k \geq N} \Gamma_{l, k}$, then for $m \geq 0$, if $I_{s}^{t} \in \mathcal{P}_{m}^{l}$, we say $I_{j}^{k} \in \mathcal{P}_{m+1}^{l}$ if $I_{j}^{k}$ is maximal (in the sense of inclusion) in $\mathcal{D}\left(I_{s}^{t}\right)$ such that

$$
\langle\nu\rangle_{I_{j}^{k}}>2\langle\nu\rangle_{I_{s}^{t}}
$$

Denote $\mathcal{P}^{l}=\cup_{m \geq 0} \mathcal{P}_{m}^{l}$ and $\pi(Q)$ is the minimal principal cube which contains $Q$. We have

$$
\begin{aligned}
\sum_{k} \sum_{l \in \mathbb{Z}} \sum_{I_{j}^{k} \in \Gamma_{l, k}} a^{k+1} \nu\left(E_{k} \cap I_{j}^{k}\right) & \leq \sum_{l \in \mathbb{Z}} c_{1} e^{-c_{2}|l|} a^{1-l} \sum_{k} \sum_{I_{j}^{k} \in \Gamma_{l, k}}\left\langle v^{\frac{1}{m}}\right\rangle_{I_{j}^{k}} \nu\left(I_{j}^{k}\right) \\
& \leq \sum_{l \in \mathbb{Z}} 2 c_{1} e^{-c_{2}|l|} a^{1-l} \sum_{I_{s}^{t} \in \mathcal{P}^{l}}\langle\nu\rangle_{I_{s}^{t}} \sum_{k, j: \pi\left(I_{j}^{k}\right)=I_{s}^{t}} v^{\frac{1}{m}}\left(I_{j}^{k}\right) \\
& \lesssim n \sum_{l \in \mathbb{Z}} c_{1} e^{-c_{2}|l|} a^{-l}\left[v^{\frac{1}{m}}\right]_{A_{\infty}} \sum_{I_{s}^{t} \in \mathcal{P}^{l}}\langle\nu\rangle_{I_{s}^{t}} v^{\frac{1}{m}}\left(I_{s}^{t}\right)
\end{aligned}
$$




$$
\begin{aligned}
& =\sum_{l \in \mathbb{Z}} c_{1} e^{-c_{2}|l|} a^{-l}\left[v^{\frac{1}{m}}\right]_{A_{\infty}} \int_{\mathbb{R}^{n}} v^{\frac{1}{m}} \sum_{I_{s}^{t} \in \mathcal{P}^{l}}\langle\nu\rangle_{I_{s}^{t}} \chi_{I_{s}^{t}} \\
& \lesssim \sum_{l \in \mathbb{Z}} c_{1} e^{-c_{2}|l|} a^{-l}\left[v^{\frac{1}{m}}\right]_{A_{\infty}}[\nu]_{A_{1}} \sum_{Q \in \mathcal{P}_{*}^{l}} \nu v^{\frac{1}{m}}(Q),
\end{aligned}
$$

where in the last step we have used the stopping criteria, i.e.,

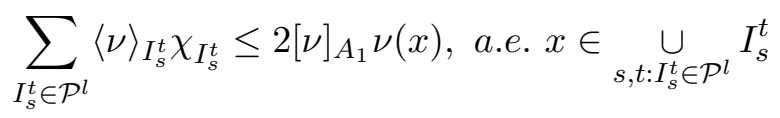

and $\mathcal{P}_{*}^{l}$ is the collection of maximal cubes (in the sense of inclusion) in $\mathcal{P}^{l}$. For fixed $Q \in \mathcal{P}_{*}^{l}$, by Lemma 3.3

$$
\begin{aligned}
\nu v^{\frac{1}{m}}(Q) & \leq C_{\nu, v}\langle\nu\rangle_{Q}\left\langle v^{\frac{1}{m}}\right\rangle_{Q}|Q| \lesssim n a^{l} C_{\nu, v} u(Q) \prod_{i=1}^{m}\left\langle f_{i}\right\rangle_{Q}^{\frac{1}{m}} \\
& \leq a^{l} C_{\nu, v}[\vec{w}]_{A_{\vec{r}}} \prod_{i=1}^{m}\left(\int_{Q} f_{i} w_{i}\right)^{\frac{1}{m}} .
\end{aligned}
$$

By the disjointness of $Q$ and Hölder's inequality, we obtain

$$
\sum_{Q \in \mathcal{P}_{*}^{l}} \nu v^{\frac{1}{m}}(Q) \lesssim a^{l} C_{\nu, v}[\vec{w}]_{A_{\overrightarrow{1}}} \prod_{i=1}^{m}\left\|f_{i}\right\|_{L^{1}\left(w_{i}\right)}^{\frac{1}{m}},
$$

and we conclude the proof.

\section{A vector-valued extension of Theorem 1.10}

Recently in [4] D. Carando, M. Mazzitelli and the second author obtained a generalization of the Marcinkiewicz-Zygmund inequalities to the context of multilinear operators. In the particular case of paraproducts, Marcinkiewicz-Zygmund inequalities were obtained by C. Benea and C. Muscalu in [1] and [2]. The results in [4] extend the previous ones in [10] and [3].

The following theorem is one of the results in [4].

Theorem 4.1 ([4]). Let $0<p, q_{1}, \ldots, q_{m}<r<2$ or $r=2$ and $0<p, q_{1}, \ldots, q_{m}<\infty$ and, for each $1 \leq i \leq m$, consider $\left\{f_{k_{i}}^{i}\right\}_{k_{i}} \subset L^{q_{i}}\left(\mu_{i}\right)$. And Let $S$ be a multilinear operator such that $S: L^{q_{1}}\left(\mu_{1}\right) \times \cdots \times L^{q_{m}}\left(\mu_{m}\right) \rightarrow L^{p, \infty}(\nu)$, then, there exists a constant $C>0$ such that

$$
\left\|\left(\sum_{k_{1}, \ldots, k_{m}}\left|S\left(f_{k_{1}}^{1}, \ldots, f_{k_{m}}^{m}\right)\right|^{r}\right)^{\frac{1}{r}}\right\|_{L^{p, \infty}(\nu)} \leq C\|S\|_{\text {weak }} \prod_{i=1}^{m}\left\|\left(\sum_{k_{i}}\left|f_{k_{i}}^{i}\right|^{r}\right)^{\frac{1}{r}}\right\|_{L^{q_{i}\left(\mu_{i}\right)}} .
$$

As a consequence of this theorem and Theorem 1.4 we obtain the following mixed weighted vector valued inequality for a multilinear Calderón-Zygmund operator $T$.

Corollary 4.2. Let $S(\vec{f})=\frac{T(\vec{f})}{v}$, where $T$ is a Calderón-Zygmund operator. Let $\vec{w}=\left(w_{1}, \ldots, w_{m}\right) \in$ $A_{\overrightarrow{1}}$ and $v \in R H_{\infty}$, or $w_{1}, \ldots, w_{m} \in A_{1}$ and $v \in A_{\infty}$. Let $\nu=w_{1}^{\frac{1}{m}} \ldots w_{m}^{\frac{1}{m}}$ and let $1<r \leq 2$. For each $1 \leq i \leq m$, consider $\left\{f_{k_{i}}^{i}\right\}_{k_{i}} \subset L^{1}\left(w_{i}\right)$. Then, there exists a constant $C>0$ such that

$$
\left\|\left(\sum_{k_{1}, \ldots, k_{m}}\left|S\left(f_{k_{1}}^{1}, \ldots, f_{k_{m}}^{m}\right)\right|^{r}\right)^{\frac{1}{r}}\right\|_{L^{\frac{1}{m}, \infty}\left(\nu v \frac{1}{m}\right)} \leq C \prod_{i=1}^{m}\left\|\left(\sum_{k_{i}}\left|f_{k_{i}}^{i}\right|^{r}\right)^{\frac{1}{r}}\right\|_{L^{1}\left(w_{i}\right)} .
$$


Observe that $S$ satisfies $S: L^{1}\left(w_{1}\right) \times \cdots \times L^{1}\left(w_{m}\right) \rightarrow L^{\frac{1}{m}, \infty}\left(\nu v^{\frac{1}{m}}\right)$, so we are under the hypothesis of Theorem 4.1 .

\section{Appendix A. Proof of Theorem 1.9}

First, as we mentioned before, that if $\vec{w}=\left(w_{1}, \ldots, w_{m}\right) \in A_{\overrightarrow{1}}$ then $\nu=w_{1}^{\frac{1}{m}} \ldots w_{m}^{\frac{1}{m}} \in A_{1}$. We will follow the ideas of [15, Theorem 1.5], and these ideas are based in previous one in [7]. Define the operator $S$ by

$$
S f(x)=\frac{M(f \nu)(x)}{\nu(x)}
$$

when $\nu(x) \neq 0$ and $S f(x)=0$ when $\nu(x)=0$. (Since $\nu \in A_{1}, \nu>0$ a.e.).

Since $\nu \in A_{1}, S$ is bounded on $L^{\infty}\left(\nu v^{\frac{1}{m}}\right)$ with constant $C=[\nu]_{A_{1}}$, that is,

$$
\|S f\|_{L^{\infty}\left(\nu v^{\frac{1}{m}}\right)} \leq[\nu]_{A_{1}}\|f\|_{L^{\infty}\left(\nu v^{\frac{1}{m}}\right)}
$$

We will now show that $S$ is bounded on $L^{p_{0}}\left(\nu v^{\frac{1}{m}}\right)$ for some $1<p_{0}<\infty$. Observe that

$$
\int_{\mathbb{R}_{n}} S f(x)^{p_{0}} \nu(x) v^{\frac{1}{m}}(x) d x=\int_{\mathbb{R}_{n}} M(f \nu)(x)^{p_{0}} \nu(x)^{1-p_{0}} v^{\frac{1}{m}}(x) d x .
$$

Since $v^{1 / m} \in A_{\infty}, v^{\frac{1}{m}} \in A_{t}$ for some $t>1$ large. Then by the $A_{p}$ factorization theorem there exist $v_{1}, v_{2} \in A_{1}$ such that $v^{\frac{1}{m}}=v_{1} v_{2}^{1-t}$; hence,

$$
\nu^{1-p_{0}} v^{\frac{1}{m}}=v_{1}\left(\nu v_{2}^{\frac{t-1}{p_{0}-1}}\right) \text {. }
$$

By Lemma 2.3 in [7] there exists $0<\varepsilon<1$, depending only on $[\nu]_{A_{1}}$, such that $\nu v_{2}^{\varepsilon} \in A_{1}$ for all $v_{2} \in A_{1}$ and $0<\varepsilon<\varepsilon_{0}$. Thus, if we let

$$
p_{0}=\frac{2(t-1)}{\varepsilon_{0}}+1
$$

then $\nu v^{\frac{1}{m}} \in A_{p_{0}}$.

By Muckenhoupt's theorem, $M$ is bounded on $L^{p_{0}}\left(\nu^{1-p_{0}} v^{\frac{1}{m}}\right)$ and therefore $S$ is bounded on $L^{p_{0}}\left(\nu v^{\frac{1}{m}}\right)$ with some constant $C_{0}$. Thus by Marcinkiewicz interpolation in the scale of Lorentz spaces, $S$ is bounded on $L^{q, 1}\left(\nu v^{\frac{1}{m}}\right)$ for all $p_{0}<q<\infty$. In particular, by [7, Proposition A.1],

$$
\|S f\|_{L^{q, 1}\left(\nu v^{\frac{1}{m}}\right)} \leq 2^{\frac{1}{q}}\left(C_{0}\left(\frac{1}{p_{0}}-\frac{1}{q}\right)+C_{1}\right)\|f\|_{L^{q, 1}\left(\nu v^{\frac{1}{m}}\right)} .
$$

Thus, for all $q \geq 2 p_{0}$ we have that $\|S f\|_{L^{q, 1}\left(\nu v^{\frac{1}{m}}\right)} \leq K_{0}\|f\|_{L^{q, 1}\left(\nu v^{\frac{1}{m}}\right)}$ with $K_{0}=4 p_{0}\left(C_{0}+C_{1}\right)$. We emphasize that the constant $K_{0}$ is valid for every $q \geq 2 p_{0}$.

Again by [7, Lemma 2.3], for every weight $W_{1} \in A_{1}$ with $\left[W_{1}\right]_{A_{1}} \leq 2 K_{0}$ there exists $0<\tilde{\varepsilon_{0}}<1$ (that depends only on $K_{0}$ ) such that $W_{1} W_{2}^{\varepsilon} \in A_{1}$ for all $W_{2} \in A_{1}$ and $0<\varepsilon<\tilde{\varepsilon_{0}}$.

Fix $0<\varepsilon<\min \left\{\tilde{\varepsilon_{0}}, \frac{1}{2 p_{0}}\right\}$ and let $r=\left(\frac{1}{\varepsilon}\right)^{\prime}$. Then $r^{\prime}>2 p_{0}$ and so $S$ is bounded on $L^{r^{\prime}, 1}\left(\nu v^{\frac{1}{m}}\right)$ with constant bounded by $K_{0}$. Now apply the Rubio de Francia algorithm to define the operator $\mathcal{R}$ on $h \in L^{r^{\prime}, 1}\left(\nu v^{\frac{1}{m}}\right), h \geq 0$, by

$$
\mathcal{R} h(x)=\sum_{k=0}^{\infty} \frac{S^{k} h(x)}{2^{k} K_{0}^{k}} .
$$

It follows immediately from this definition that:

- $h(x) \leq \mathcal{R} h(x)$;

- $\|\mathcal{R} h\|_{L^{r^{\prime}, 1\left(\nu v^{\frac{1}{m}}\right)}} \leq 2\|h\|_{L^{r^{\prime}, 1}\left(\nu v^{\frac{1}{m}}\right)}$; 
- $S(\mathcal{R} h)(x) \leq 2 K_{0} \mathcal{R} h(x)$.

In particular, it follows from the last item and the definition of $S$ that $\mathcal{R} h \nu \in A_{1}$ with $[\mathcal{R} h \nu]_{A_{1}} \leq$ $2 K_{0}$. Let $W_{1}=\mathcal{R} h \nu$ and $W_{2}=v_{1} \in A_{1}$. Then $W_{1} W_{2}^{\varepsilon} \in A_{1}$. Hence, $\mathcal{R} h \nu v^{\frac{1}{m r^{\prime}}} \in A_{1} \subset A_{\infty}$.

Then,

$$
\begin{aligned}
\left\|\frac{T(\vec{f})}{v}\right\|_{L^{\frac{1}{m}, \infty}\left(\nu v^{\left.\frac{1}{m}\right)}\right.}^{\frac{1}{m r}} & =\sup _{\lambda>0} \lambda^{\frac{1}{m r}}\left(\nu v^{\frac{1}{m}}\left\{x \in \mathbb{R}^{n}:\left|\frac{T(\vec{f})(x)}{v(x)}\right|>\lambda\right\}\right)^{\frac{1}{r}} \\
& =\sup _{\lambda>0} \lambda^{\frac{1}{m r}}\left(\nu v^{\frac{1}{m}}\left\{x \in \mathbb{R}^{n}:\left|\frac{T(\vec{f})(x)}{v(x)}\right|^{\frac{1}{m r}}>\lambda^{\frac{1}{m r}}\right\}\right)^{\frac{1}{r}} \\
& =\sup _{t>0} t\left(\nu v^{\frac{1}{m}}\left\{x \in \mathbb{R}^{n}:\left|\frac{T(\vec{f})(x)}{v(x)}\right|^{\frac{1}{m r}}>t\right\}\right)^{\frac{1}{r}} \\
& =\left.\left\|\left(\frac{T(\vec{f})}{v}\right)^{\frac{1}{m r}}\right\|_{L^{r, \infty}\left(\nu v^{\frac{1}{m}}\right)}\left|\int_{\mathbb{R}^{n}}\right| \frac{T(\vec{f})(x)}{v(x)}\right|^{\frac{1}{m r}} h(x) \nu(x) v^{\frac{1}{m}}(x) d x \mid \\
& =\left.\sup _{h \in L^{r^{\prime}, 1}\left(\nu v^{\frac{1}{m}}\right):\|h\|_{\left.L^{r^{\prime}, 1(\nu v} \frac{1}{m}\right)}=1} \sup _{h \in L^{r^{\prime}, 1}\left(\nu v^{\frac{1}{m}}\right):\|h\|_{\left.L^{r^{\prime}, 1(\nu v} \frac{1}{m}\right)}=1}\left|\int_{\mathbb{R}^{n}}\right| T(\vec{f})(x)\right|^{\frac{1}{m r}} h(x) \nu(x) v^{\frac{1}{m r^{\prime}}}(x) d x \mid .
\end{aligned}
$$

Before finishing we recall the following fact, which was proved in [13] (see Corollary 3.8 there), if $w \in A_{\infty}$ and $s>0$ then

$$
\int_{\mathbb{R}^{n}}|T(\vec{f})(x)|^{s} w(x) d x \leq C \int_{\mathbb{R}^{n}} \mathcal{M}(\vec{f})(x)^{s} w(x) d x .
$$

From the definition of $\mathcal{R} h(x)$, the last inequality and Hölder's inequality

$$
\begin{aligned}
\int_{\mathbb{R}^{n}}|T(\vec{f})(x)|^{\frac{1}{m r}} h(x) \nu(x) v^{\frac{1}{m r^{\prime}}}(x) d x & \leq \int_{\mathbb{R}^{n}}|T(\vec{f})(x)|^{\frac{1}{m r}} \mathcal{R} h(x) \nu(x) v^{\frac{1}{m r^{\prime}}}(x) d x \\
& \leq \int_{\mathbb{R}^{n}} \mathcal{M}(\vec{f})(x)^{\frac{1}{m r}} \mathcal{R} h(x) \nu(x) v^{\frac{1}{m r^{\prime}}}(x) d x \\
& =C \int_{\mathbb{R}^{n}}\left(\frac{\mathcal{M}(\vec{f})(x)}{v(x)}\right)^{\frac{1}{m r}} \mathcal{R} h(x) \nu(x) v^{\frac{1}{m}}(x) d x \\
& \leq C\left\|\left(\frac{\mathcal{M}(\vec{f})}{v}\right)^{\frac{1}{m r}}\right\|_{L^{r, \infty}\left(\nu v^{\frac{1}{m}}\right)}\|\mathcal{R} h\|_{L^{r^{\prime}, 1}\left(\nu v^{\frac{1}{m}}\right)} \\
& \leq 2 C\left\|\frac{\mathcal{M}(\vec{f})}{v}\right\|_{L^{\frac{1}{m}, \infty}\left(\nu v^{\frac{1}{m}}\right)}^{\frac{1}{m r}}\|h\|_{L^{r^{\prime}, 1}\left(\nu v^{\frac{1}{m}}\right)} \\
& =2 C\left\|\frac{\mathcal{M}(\vec{f})}{v}\right\|_{L^{\frac{1}{m r}}}^{\left.\frac{1}{m}, \infty v^{\frac{1}{m}}\right)}
\end{aligned}
$$

So we have that

$$
\left\|\frac{T(\vec{f})}{v}\right\|_{L^{\frac{1}{m}, \infty}\left(\nu v^{\frac{1}{m}}\right)}^{\frac{1}{m^{2}}} \leq C\left\|\frac{\mathcal{M}(\vec{f})}{v}\right\|_{L^{\frac{1}{m}, \infty}\left(\nu v^{\frac{1}{m}}\right)}^{\frac{1}{m r}} .
$$

\section{REFERENCES}

[1] C. Benea and C. Muscalu, Multiple vector valued inequalities via the helicoidal method. Anal. PDE, 9: 19311988, 2016. 
[2] C. Benea and C. Muscalu, Quasi-Banach valued inequalities via the helicoidal method. Preprint, arXiv: 1609.01090 .

[3] F. Bombal, D. Pérez-García and I. Villanueva, Multilinear extensions of Grothendieck's theorem. Quart. J. Math., 55(4): 441-450, 2004.

[4] D. Carando, M. Mazzitelli and S. Ombrosi, Multilinear Marcinkiewicz-Zygmund inequalities, arXiv:1611.08284 (2016).

[5] R.R. Coifman and Y. Meyer, On commutators of singular integrals and bilinear singular integrals, Trans. Amer. Math. Soc. 212 (1975), 315331.

[6] R.R. Coifman and Y. Meyer, Commutateurs dintégrales singulires et opérateurs multilineaires, Ann. Inst. Fourier (Grenoble) 28 (1978), no. 3, 177202.

[7] D. Cruz-Uribe, J.M. Martell and C. Pérez, Weighted weak-type inequalities and a conjecture of Sawyer Int. Math. Res. Not., 30 (2005), 1849-1871.

[8] D. Cruz-Uribe and C. J. Neugebauer, The structure of the reverse Hlder classes Trans. Amer. Math. Soc. 347 (1995), 2941-2960

[9] J. García-Cuerva and J.L. Rubio de Francia, Weighted norm inequalities and related topics. North-Holland Math. Stud. 116, North-Holland, 1985.

[10] L. Grafakos and J.M. Martell, Extrapolation of weighted norm inequalities for multivariable operators and applications. J. Geom. Anal., 14(1): 19-46, 2004.

[11] L. Grafakos and R.H. Torres, Multilinear Calderón-Zygmund theory, Adv. Math. 165 (2002), no.1, 124164.

[12] L. Grafakos and R.H. Torres, Maximal operator and weighted norm inequalities for multilinear singular integrals, Indiana Univ. Math. J., 51 (2002), no. 5, 12611276.

[13] A. Lerner, S. Ombrosi, C. Pérez, R. H. Torres and R. Trujillo-Gonzales, New maximal functions and multiple weights for the multilinear Calderón-Zygmund theory Adv. Math., 220 (2009) no. 4, 1222-1264.

[14] K. Li, S. Ombrosi and C. Pérez, Proof of an extension of E. Sawyer's conjecture about weighted mixed weak-type estimates, arXiv:1703.01530 (2017).

[15] S. Ombrosi and C. Pérez, Mixed weak type estimates: Examples and counterexamples related to a problem of E. Sawyer, Colloquium Mathematicum, 145 (2016), 259-272.

[16] S. Ombrosi, C. Pérez and J. Recchi, Quantitative weighted mixed weak-type inequalities for classical operators, Indiana Univ. Math. J., 65(2016) 615-640.

[17] C. Pérez and R.H. Torres, Sharp maximal function estimates for multilinear singular integrals, Contemp. Math., 320 (2003), 323331.

[18] E. Sawyer, A weighted weak type inequality for the maximal function Proc. Amer. Math. Soc. 93 (1985), 610-614.

(K. Li) BCAM, Basque Center for Applied Mathematics, Mazarredo, 14. 48009 Bilbao Basque COUNTRY, SPAIN

E-mail address: kli@bcamath.org

(S. Ombrosi) Departamento de Matemática, Universidad Nacional del Sur, Bahía Blanca, 8000, Argentina

E-mail address: sombrosi@uns.edu.ar

(B. Picardi) Departamento de Matemática, Universidad Nacional del Sur, Bahía Blanca, 8000, Argentina

E-mail address: belen.picardi@uns.edu.ar 